\title{
Modeling and Control of Discrete-Event Systems with Partial Non-Determinism using Petri Nets
}

\section{František Čapkovič}

Institute of Informatics, Slovak Academy of Sciences, Dúbravská cesta 9, 84507

Bratislava, Slovakia, e-mail: Frantisek.Capkovic@savba.sk

\begin{abstract}
Discrete-Event Systems (DES) are systems that are discrete in nature. A next state of DES depends on the actual state and on the occurrence of a discrete event. DES are often modeled and controlled by Petri Nets $(P N)$ of different kinds (placeltransition PN, timed PN, etc.). However, not always real DES, are purely deterministic. In such cases, the $P N$-based model contains some uncontrollable and/or unobservable transitions or unmeasurable/unobservable places. In order to control DES with the partial nondeterminism, special kinds of $P N$ and control methods/procedures have to be used. That's just it - applications of Interpreted PN (IPN) and Labeled PN (LbPN) for modeling analyzing and control of DES are investigated here.
\end{abstract}

Keywords: control; discrete-event systems; modeling; non-determinism; Petri nets

\section{Introduction}

Discrete-Event Systems (DES) are systems where a next state depends only on the actual state and on the occurrence of a discrete event. It can be said that DES are systems discrete in nature. In other words, such a system persists in a state until it is not forced to change its state in consequence of a discrete event occurrence. Many kinds of real systems in practice have the character of DES - e.g. flexible manufacturing systems, robotized working cells, discrete production lines, some kinds of transport systems, communication systems etc. Here, discrete events have the character of starting or ending of particular operations, synchronization of several operations etc., but also external influences. The simple abstract illustrative example presenting the causal relation between the system states $x_{i}$, $i=1, \ldots, 6$ and the occurrence of discrete events $u_{j}, j=1, \ldots, 5$ is given in Figure 1 . Here, the system response on the discrete event sequence $\left\{u_{1}, u_{2}, u_{3}, u_{4}, u_{5}\right\}$ is the sequence of states $\left\{x_{3}, x_{1}, x_{2}, x_{5}, x_{4}, x_{6}\right\}$, where $x_{3}$ is the initial state. A view on DES and their history is presented in [6]. The global overview on modeling, analysis and control of DES is given in [7]. 
DES are frequently modeled by means of different kinds of Petri Nets (PN) like Place/Transition PN (P/T PN) - alias ordinary PN, timed PN, hybrid PN, colored $\mathrm{PN}$, etc. However, DES are not always purely deterministic. They can be partially nondeterministic. Some newer, specific kinds of PN, like Labeled PN (LbPN), Interpreted PN (IPN) are able to deal better with modeling, analyzing and control of DES with non-determinism like those mentioned above.

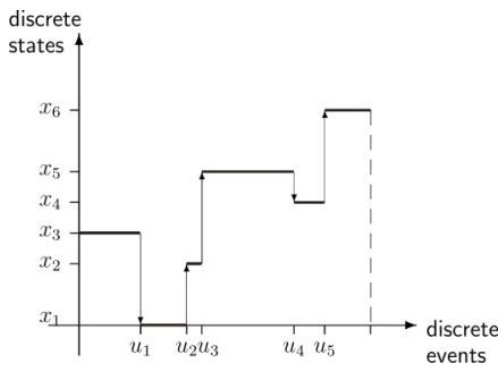

Figure 1

The evolution of states in DES

Non-determinism in DES can be caused, in principle, by means of two different factors: (i) occurrence of silent events, that cause a change in the DES state and they are not observable by any external observer; (ii) indistinguishable events, whose occurrence at a given DES state yields two or more new states.

\subsection{Petri Nets}

Essentials of PN were defined by C.A. Petri in his PhD thesis [1] written in German. Later, some novels were brought in [2] - [4]. Nowadays, PN represent a huge variety of PN kinds and methods of their mathematical modeling, analysis and control. The very good survey of PN evolution since [1] is presented in [5].

In principle, P/T PN are bipartite directed graphs with two kinds of nodes and two kinds of edges. Nodes $p_{i}, i=1, \ldots, n$, are named as places and represent elementary states of particular operations in modeled DES. Let $P=\left\{p_{1}, \ldots, p_{n}\right\}$ is the set of places. Edges $t_{j}, j=1, \ldots, m$, are named as transitions and model the discrete events. Let $T=\left\{t_{1}, \ldots, t_{m}\right\}$ is the set of transitions. The causality among the states and events is expressed by the sets $F \subseteq P \times \mathrm{T}, G \subseteq T \times P$ representing, respectively, the incidence of directed arcs from places to transitions and from transitions to places. Then, the structure of $\mathrm{P} / \mathrm{T} \mathrm{PN}$ can be formally expressed by the following quadruplet

$<P, T, F, G>$

The P/T PN have also their dynamics expressing the evolution of the state in the steps $k=0,1, \ldots$, which can be described by the restricted discrete linear vector state equation as follows 
$\mathbf{x}_{k+1}=\mathbf{x}_{k}+\mathbf{B} \cdot \mathbf{u}_{k}, k=0,1, \ldots$

F. $\mathbf{u}_{k} \leq \mathbf{x}_{k}$

Where,

$\mathbf{x}_{k}=\left(x_{p_{1}}, x_{p_{2}}, \ldots, x_{p_{n}}\right)^{T}$ is the state vector with entries $x_{p_{i}} \in\{0,1, \ldots, \infty\}$ being the states of particular places $p_{i}, i=1, \ldots, n$, in the step $k$, namely $x_{p_{i}}$ represents the actual number of tokens in the place $p_{i}$. The vector $\mathbf{x}_{0}$ is the initial state vector. There are three basic kinds of places: (i) operation places (e.g. in models of flexible manufacturing systems (FMS) they reflect the progress of processing parts in particular steps) being not marked in the initial state, (ii) places representing presence of fix resources (e.g. machine tools, robots, conveyors, buffers, etc. in FMS) being marked in the initial state, (iii) places representing variable resources (e.g. availability of raw materials, semi-products in FMS).

In PT/PN places can be observable/measurable. In IPN and LbPN some places may be unobservable/unmeasurable.

$\mathbf{u}_{k}=\left(u_{t_{1}}, u_{t_{2}}, \ldots, u_{t_{m}}\right)^{T}$ is the control vector with entries $u_{t_{j}} \in\{0,1\}$ being the states of particular transitions $t_{j}, j=1, \ldots, m$, in the step $k$. They can be disabled or enabled - namely, when the transition $t_{j}$ is disabled (i.e. it cannot be fired) then $u_{t_{j}}=0$, otherwise it is enabled (i.e. it may be, but not always has to be, fired) and then $u_{t_{j}}=1$. In P/T PN enabled transitions represent the occurrence of discrete events which can be observable and/or controllable. However, in IPN and LbPN some events may be spontaneous, i.e. the transitions which model them are uncontrollable and/or unobservable.

$\mathbf{B}=\mathbf{G}^{T}-\mathbf{F}$ is the structural matrix with $\mathbf{G}$ being the incidence matrix corresponding to the set $G$ and $\mathbf{F}$ being the incidence matrix corresponding to the set $F$. The term $\mathbf{x}_{0}$ is the initial state vector.

Starting from $\mathbf{x}_{0}$ and firing an enabled transition the next state $\mathbf{x}_{1}$ can be reached. The reachability tree (RT) expresses all possible branches of the development of the system (1) - (2). When all transitions are observable and controllable and all places are measurable/observable, the system development can be controlled without greater problems. For example for a firing sequence of transitions $t_{a}, t_{b}$, $\ldots, t_{c}$, the state trajectory will be the following $\mathbf{x}_{0} \stackrel{u_{t_{a}}}{\longrightarrow} \mathbf{x}_{1} \stackrel{u_{t_{b}}}{\longrightarrow} \ldots \mathbf{x}_{\mathrm{k}-1} \stackrel{u_{t_{c}}}{\rightarrow} \mathbf{x}_{\mathrm{k}}$. Such trajectories represent branches in RT. RT is a standard tree with nodes expressing state vectors $\mathbf{x}_{k}, k=0, \ldots, K$, where $K$ is the global number of states. $K$ may be infinite too. The states create the state space - the set $\mathcal{R}=\left\{\mathbf{x}_{0}, \mathbf{x}_{1}, \ldots, \mathbf{x}_{\mathrm{k}}\right\}$ of reachable states. The edges of RT symbolize the PN transitions.

In the PN theory the term marking $M$ is used instead the term state $\mathbf{x}$. Then, the above introduced trajectory in such a perception and symbolism has the form $M_{0}\left[u_{t_{a}}>\Rightarrow M_{1}\left[u_{t_{b}}>\Rightarrow \cdots \Rightarrow M_{k-1}\left[u_{t_{c}}>\Rightarrow M_{k}\right.\right.\right.$ or $M_{0}\left[U>\Rightarrow M_{k}\right.$, where the set $U=$ $\left\{u_{t_{a}}, u_{t_{b}}, u_{t_{c}}\right\}$. 
Unfortunately, in PN models of real DES the situation is not so simple because of the above mentioned silent and indistinguishable transitions. From the DES control point of view it is necessary a deeper view. Thus, it is needful to speak about uncontrollable and/or unobservable transitions and moreover, even about unmeasurable/unobservable places. Namely, when a controller is not allowed to affect some of transitions, the transitions become uncontrollable. Their firing cannot be either inhibited or allowed by any external action. A transition is named to be unobservable when its firing cannot be directly detected or measured. Unobservable transitions model internal events being not observable from outside. Any unobservable transition is implicitly uncontrollable.

Analogically, the state (marking) of an unmeasurable/unobservable place cannot be detected or measured. In such a case the set of observable places is reduced. Thus, the reduced number of entries creates the output vector. It means that an output equation has to be added to the PN model (1), (2).

Consequently, in order to model and control a real DES, new approaches to PNbased modeling and control have to be found. LbPN and IPN are able to help us on such a way.

\subsection{A Simple Example of P/T PN}

To illustrate the P/T PN structure and dynamics let us introduce a simple example. In Figure 2 the PN structure is presented while in Figure 3a, Figure 3b, respectively, the corresponding $\mathrm{RT}$ and $\mathrm{RG}$.

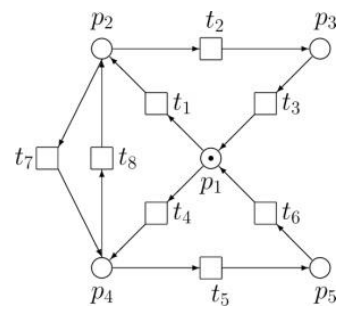

Figure 2

A simple P/T PN

The incidence matrices representing the PN structure are as follows

$$
\mathbf{F}=\left(\begin{array}{llllllll}
1 & 0 & 0 & 1 & 0 & 0 & 0 & 0 \\
0 & 1 & 0 & 0 & 0 & 0 & 1 & 0 \\
0 & 0 & 1 & 0 & 0 & 0 & 0 & 0 \\
0 & 0 & 0 & 0 & 1 & 0 & 0 & 1 \\
0 & 0 & 0 & 0 & 0 & 1 & 0 & 0
\end{array}\right) ; \mathbf{G}^{T}=\left(\begin{array}{llllllll}
0 & 0 & 1 & 0 & 0 & 1 & 0 & 0 \\
1 & 0 & 0 & 0 & 0 & 0 & 0 & 1 \\
0 & 1 & 0 & 0 & 0 & 0 & 0 & 0 \\
0 & 0 & 0 & 1 & 0 & 0 & 1 & 0 \\
0 & 0 & 0 & 0 & 1 & 0 & 0 & 0
\end{array}\right)
$$

and the initial state is

$$
\mathbf{x}_{0}=\left(\begin{array}{lllll}
1 & 0 & 0 & 0 & 0
\end{array}\right)^{T} .
$$


By means of the model of dynamics development represented by (1) - (2) other four following states can be reached from the initial state
$\mathbf{x}_{1}=\left(\begin{array}{llll}0 & 1 & 0 & 0\end{array}\right.$
$0)^{T} ; \mathbf{x}_{2}=(0$
$\begin{array}{lll}0 & 0 & 1\end{array}$
$0)^{T} ; \mathbf{x}_{3}=(0$
0
$\begin{array}{ll}0 & 0)^{T} \text { and }\end{array}$
$\mathbf{x}_{4}=\left(\begin{array}{lllll}0 & 0 & 0 & 0 & 1\end{array}\right)^{T}$

Thus, the space of reachable states $\mathcal{R}=\left\{\mathbf{x}_{0}, \mathbf{x}_{1}, \mathbf{x}_{2}, \mathbf{x}_{3}, \mathbf{x}_{4}\right\}$. Corresponding RT represents causal relations among states, i.e. among nodes being the state vectors. Such a transit among states is ensured by particular transitions. For drawing P/T $\mathrm{PN}$, analyzing their properties and drawing RT different kinds of graphical simulators can be used. Thus RT of this P/T PN is given in Figure 3. This RT and next RTs are outputs of the graphical simulator of PN. It affects their quality. The corresponding reachability graph (RG) arises by connecting RT nodes with the same name into one node - see Figure 3a. The adjacency matrices in the form A or $\mathbf{A}_{d}$ (expressing dynamic entries) are the same for both RT and RG, namely

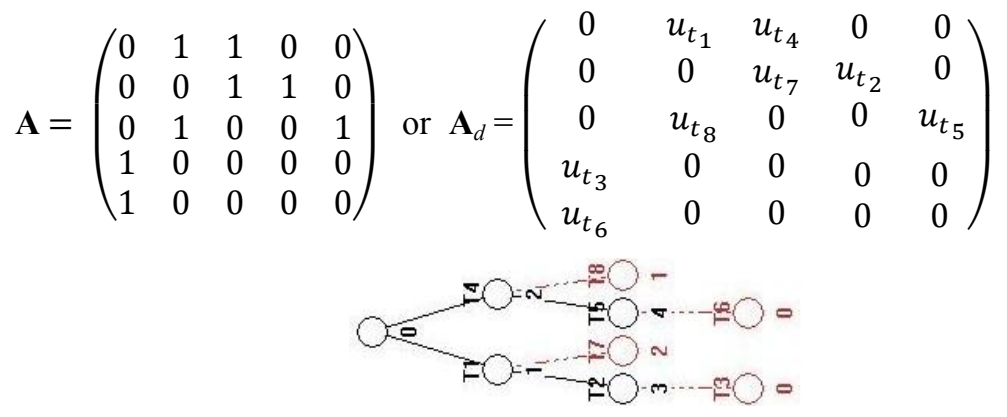

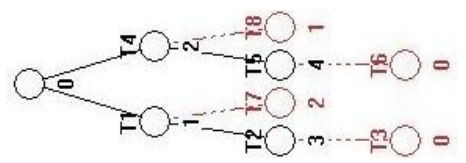

Figure $3 \mathrm{a}$

The corresponding RT (obtained from the graphic simulator of P/T PN)

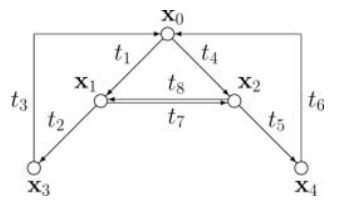

Figure $3 b$

The reachability graph RG corresponding to RT

\subsection{The Paper Organization}

While in this Section 1 the PN were defined, in the next Section 2 definitions of LbPN and IPN will be introduced. The particularities of such kinds of PN will be pointed out and shortly illustrated on corresponding examples.

The Section 3 will bring the core issues of the paper concerning the usage of IPN and LbPN in DES modeling and control. Just practical examples of control of real DES working in nondeterministic conditions by means of such kinds of PN represent the main contributions of this paper. Namely, the IPN and LbPN models 
of such kinds of complicated DES, containing uncontrollable/unobservable transitions and unmeasurable/unobservable places, are able in sequence to show (i) how to describe the non-determinism of such systems; (ii) how they make possible to analyze the nondeterministic DES; (iii) how to synthesize approaches for the control of such DES; (iv) how the control of such DES can be performed.

In the section Conclusion the global evaluation of the paper contributions is introduced.

Finally, the list of References is included.

\section{Labeled and Interpreted Petri Nets}

LbPN and IPN are purpose-built extended forms of P/T PN. They make possible to deal with the PN containing uncontrollable and/or unobservable transitions and unmeasurable/unobservable places. There are many definitions of LbPN - e.g. in [8]-[12], [14]-[16] - as well as of IPN - e.g. in [13]. It is necessary to say that the primal definition of IPN arose in the Mexican group in CINVESTAV Unidad Guadalajara, where many other papers about IPN besides [13] were written.

\subsection{Labeled Petri Nets}

In case of LbPN the term net $N$ means the P/T PN structure. Besides $N$ there are additional attributes - the alphabet $\mathcal{L}=L \cup \varepsilon$, where $L$ expresses the observable events and $\varepsilon$ represent unobservable events; the labeling function $\ell: T \rightarrow \mathcal{L}$ assigning events to transitions; the set of reachable states $\mathcal{R}$; the set of finite states $F_{x} \in \mathcal{R}$ (i.e. $F_{x} \subset \mathcal{R}$ ). It means that LbPN can be formally expressed as the following quintuple

$<N, \mathcal{L}, \ell, \mathbf{x}_{0}, F_{x}>$

There exist three kinds of labeled functions in LbPN, namely: (i) free labeling, when $\ell$ is a one-to-one mapping; (ii) $\lambda$-free labeling, when two or more transitions share the same label; (iii) arbitrary labeling, when $\ell: T \rightarrow \mathcal{L} \cup\{\lambda\}$ with $\lambda$ being an empty string.

Although this paper does not deal with the problem of LbPN diagnosability, it is necessary to say that (freely interpreted) the diagnosability of an LbPN, with unobservable transitions, implies [17] that each occurrence of a fault can be detected after a finite number of transition firings.

\subsubsection{Illustrative Example}

Consider the simple LbPN given in Figure 4. The transitions are labeled by assigned events $a, b$. The RT of this net is displayed in Figure 5. 


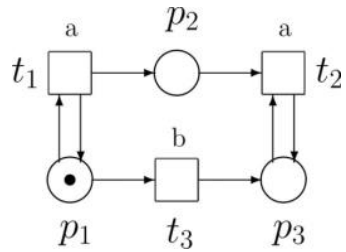

Figure 4

A simple LbPN

Here, in the Figure 4, two kinds of mappings can be seen - namely free (at the transitions $t_{1}$ and $t_{3}$ ) and $\lambda$-free (at the transitions $t_{1}$ and $t_{2}$ ). The RT of the LbPN is given in Figure 5. As we see there, the RT contains ambiguities. Namely, there are two cycles there, pointing out to two ambiguous states $\mathbf{x}_{1}$ and $\mathbf{x}_{3}$. Namely, the set $\mathcal{R}$ consists of the following states

$\mathbf{x}_{0}=\left(\begin{array}{lll}1 & 0 & 0\end{array}\right)^{T} ; \mathbf{x}_{1}=\left(\begin{array}{lll}1 & \omega & 1\end{array}\right)^{T} ; \mathbf{x}_{2}=\left(\begin{array}{lll}0 & 0 & 1\end{array}\right)^{T} ; \mathbf{x}_{3}=\left(\begin{array}{lll}0 & \omega & 1\end{array}\right)^{T}$

where $\omega$ corresponds to the so called self-loops, i.e. when for $p \in P, t \in T,\{(p, t)$ $\in B=(t, p) \in B\}$. Here the set $B=F \cup G$ corresponds to the matrix $\mathbf{B}$.

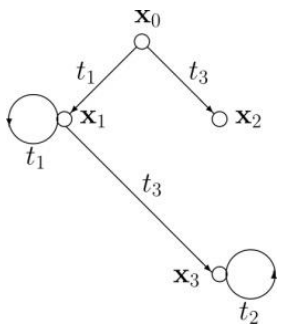

Figure 5

The corresponding RT

In our case the loops are represented by means of the pairs $\left(p_{1}, t_{1}\right)$ and $\left(p_{3}, t_{2}\right)$. The analysis of RT, more precisely the coverability graph (CG) in this case, is much more complicated than the previous analysis of RT in Figure 3. Namely, the deploying of such CG to the labeled reachability graph (LbRG) leads to the infinite chain given in Figure 6. On the basis of such RT we can analyze the LbPN given on Figure 4 by means of the alphabet $\mathcal{L}$. When all events are observable (i.e. when no $\varepsilon$ occurs in $\mathcal{L}$ ) the situation is relatively simple.

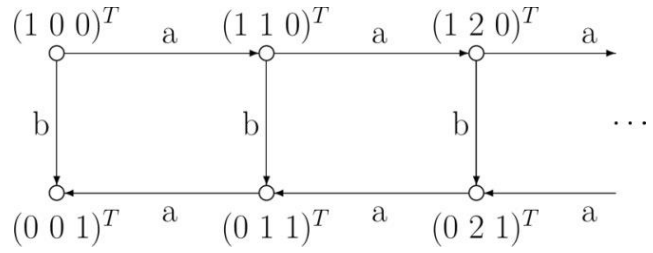

Figure 6

The CG deployed to LbRG 
When we start from the initial state $\mathbf{x}_{0}=\left(\begin{array}{lll}1 & 0 & 0\end{array}\right)^{T}$ by firing $t_{3}$ the system development will be finished in the state $\mathbf{x}_{2}=\left(\begin{array}{lll}0 & 0 & 1\end{array}\right)^{T}$. Suppose that $\mathbf{x}_{2}$ is the desired final state (i.e. $\mathbf{x}_{2} \in F_{x} \subset \mathcal{R}$ ). The final state can be reached from the initial, by the following firing sequences: $\{b\},\{a, b, a\},\{a, a, b, a, a\},\{a \mathrm{a} a \mathrm{~b}$ a a $\mathrm{a}\}$, etc. It means that formally written $L=\left\{\mathrm{a}^{\mathrm{m}} \mathrm{b} \mathrm{a}^{\mathrm{m}} \mid \mathrm{m} \geq 0\right\}$. When an unobservable event $\varepsilon$ occurs in the alphabet $\mathcal{L}$, i.e. when an unobservable transition occurs in the PN, the situation changes. During control synthesis it is necessary to deal with them.

\subsection{Interpreted Petri Nets}

In case of IPN the symbol $N$ also means the P/T PN. Besides $N$ there are the following additions here - the input alphabet $\Sigma=\left\{\alpha_{1}, \alpha_{2}, \ldots, \alpha_{r}\right\}$ with the input symbols $\alpha_{i}, i=1, \ldots, r$; the output alphabet $\Phi=\left\{\delta_{1}, \delta_{2}, \ldots, \delta_{s}\right\}$ with the output symbols $\delta_{j}, j=1, \ldots, s$; the labeling function of transitions $\lambda=T \rightarrow \Sigma \cup\{\varepsilon\}$ which assigns to each transition either $\alpha_{i} \in \Sigma$ or an internal event. Here, a constraint asking that a unique label is assigned to each transition, i.e. $\forall t_{j}, t_{k} \in T$, $j \neq k$ if $\forall p_{i} \mathbf{F}\left(p_{i}, t_{j}\right)=\mathbf{F}\left(p_{i}, t_{k}\right) \neq 0$ and both $\lambda\left(t_{j}\right) \neq \varepsilon, \lambda\left(t_{k}\right) \neq \varepsilon$ then $\lambda\left(t_{j}\right) \neq$ $\lambda\left(t_{k}\right)$ has to be valid; the labeling function of places $\Psi=P \rightarrow \Phi \cup\{\varepsilon\}$ which assigns to each place either output symbol $\delta_{i} \in \Phi$ or $\varepsilon$ being a null output signal; the output function $\mathbf{C}: \mathcal{R}$ (IPN, $\left.\mathbf{x}_{0}\right) \rightarrow \mathbb{Z}_{\geq 0}^{q \times n}$ (here, $\mathbb{Z}$ is the set of integers) with $q$ $\in \mathbb{Z}_{>0}$ being the positive integer expressing the number of available output signals and $n=|P|$ being the number of places, is the $(q \times n)$-dimensional matrix of integers assigning the output vector to each marking $\mathcal{R}$ (IPN, $\mathbf{x}_{0}$ ). Thus, the ( $q \times$ $n$ )-dimensional matrix $\mathbf{C}$ represents a relation between output vectors and state vectors of IPN. IPN can be formally expressed by means of the following sextuple

$<\mathrm{N}, \Sigma, \Phi, \lambda, \Psi, \mathbf{C}>$

Because the definition may seem too complicated, an illustrative example should be introduced for explanation.

\subsubsection{Illustrative Example}

In Figure 7 the simple example of IPN is displayed. There are two uncontrollable/unobservable places $t_{3}, t_{6}$ and two unmeasurable /unobservable places $\mathrm{p}_{3}$ and $\mathrm{p}_{5}$. Here, the input alphabet $\boldsymbol{\Sigma}=\left\{\boldsymbol{\alpha}_{1}, \ldots, \boldsymbol{\alpha}_{\mathbf{4}}\right\}$, correspond to controllable transitions $\left\{t_{1}, t_{2}, t_{4}, t_{5}\right\}$ assigned by the function $\lambda$. By the same function the symbol $\boldsymbol{\varepsilon}$ is assigned to the uncontrollable/unobservable transitions $\left\{t_{3}, t_{6}\right\}$. The measurable places are $\left\{p_{1}, p_{2}, p_{4}, p_{6}\right\}$. They correspond with the output alphabet $\boldsymbol{\Phi}=\left\{\boldsymbol{\delta}_{\mathbf{1}}, \ldots, \boldsymbol{\delta}_{\mathbf{4}}\right\}$. The symbol $\boldsymbol{\varepsilon}$ is assigned to the unmeasurable/unobservable places $\left\{p_{3}, p_{5}\right\}$. They yield the null output signal. The places $\left\{p_{7}, p_{8}\right\}$ have the fixed marking because of the self-loops. Therefore, the following output equation

$\mathbf{y}_{k}=\mathbf{C} . \mathbf{x}_{k}, k=0,1, \ldots, K$ 
extends the model (1), (2). In case of this illustrative IPN it means that

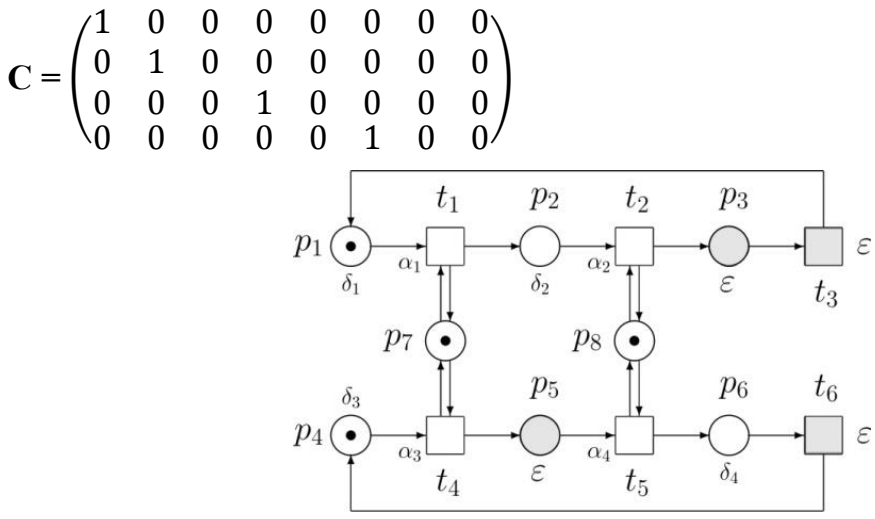

Figure 7

The IPN with two uncontrollable transitions and two unmeasurable/unobservable places

\section{Interpreted and Labeled Petri Nets in DES Control}

As it was mentioned above, PN are used for modeling, analyzing and control of DES. PN models of real systems may comprehend uncontrollable and/or unobservable transitions and unmeasurable/unobservable places. Therefore, the set of transitions $T$ consists of two subsets $T=T_{c} \cup T_{u}$, where $T_{\mathrm{c}}$ includes all controllable transitions and $T_{u}$ involves all uncontrollable transitions. Some of controllable transitions detecting their firing are named as the transition sensors. The set of places $P$ also consists of two subsets $P=P_{m} \cup P_{u m}$, where $P_{m}$ includes all measurable places and $P_{u m}$ involves all unmeasurable/unobservable places. Some places from $P_{m}$ may represent the so called place sensors - see e.g. $\left\{p_{1}, p_{2}\right.$, $\left.p_{4}, p_{6}\right\}$ in Figure 7 . Such places creates the $(q \times n)$-dimensional output vector $\mathbf{y}_{k}$ in (10).

\subsection{The IPN View on the Problem of Control}

From the IPN point of view it is possible to utilize a general view. Consider the following simple introduction into the principle of the IPN based control.

\subsubsection{The Basic Principle of the IPN-based Control}

Consider a controlled segment of a plant. Its IPN model is displayed in Figure 8. There is (i) a control specification represented by the subnet $\left\{p_{4} ; t_{3}\right\}$ and; (ii) the model of a segment of the plant (to be controlled) represented by the subnet $\left\{p_{1}\right.$, $\left.p_{2}, p_{3} ; t_{1}, t_{2}\right\}$. The place $p_{2}$ is unmeasurable/unobservable and $t_{2}$ is uncontrollable/unobservable. 


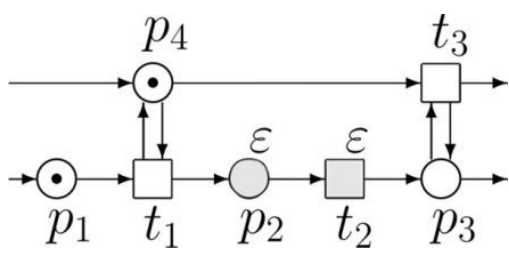

Figure 8

The principle of IPN-based control

Here, on the one hand, the controllable transition $t_{1}$ representing a discrete event in the plant is enabled by means of the self-loop with the sensor place $p_{4}$ of the control specification. On the other hand, the measurable place (sensor) $p_{3}$ of the plant becomes active by means of the self-loop with $t_{3}$. In such a way the uncontrollable transition $t_{2}$ (being an internal spontaneous discrete event) and the unmeasurable/unobservable place $p_{2}$ are bypassed. Thus, the transition $t_{2}$ (labeled practically by $\varepsilon$ ) can spontaneously fire or not. No interference from outside to $t_{2}$ is possible. In spite of this, the situation (i.e. the system dynamics development) is under the control. The RT of the IPN has only one branch $-\mathbf{x}_{0} \stackrel{u_{t_{1}}}{\longrightarrow} \mathbf{x}_{1} \stackrel{u_{t_{2}}}{\longrightarrow} \mathbf{x}_{2} \stackrel{u_{t_{3}}}{\longrightarrow} \mathbf{x}_{3}$. In spite of this the control system is not able to force transition $t_{2}$ directly. However, it is pent inside the control loop and it cannot influence another parts of plant. Of course, the control specifications have to respect the prescribed manufacturing system technology (i.e. the technological process).

The best way how to present the approaches to the real DES control are examples.

\subsection{Example 1}

Consider the example of the real DES given in Figure 9 being a complex FMS. Here, activities of two automatically guided vehicles AGV 1, AGV 2 are modeled. The activity of AGV 2 is modeled by means of the upper PN subnet $\left\{p_{1}, p_{2}, p_{3}, p_{4}\right.$; $\left.t_{2}, t_{3}, t_{4}, t_{5}\right\}$ and the activity of AGV 1 is modeled by means of the lower PN subnet $\left\{p_{6}, p_{7}, p_{8}, p_{9} ; t_{6}, t_{7}, t_{8}\right\}$. AGVs cooperate in FMS in such a way that: (i) the role of AGV 2 is to carry two different parts A, B from two input conveyors (each of them feeds the corresponding kind of parts) into a transship center Transfer; (ii) the role of AGV 1 is to carry these products to a robot R which put the parts (by means of its gripper) on a pressure plate of a compactor machine where the parts are pressed down altogether into a final product $\mathrm{C}$ (i.e. $\mathrm{C}=\mathrm{A}+\mathrm{B}$ ). The $\mathrm{PN}$ model of the FMS to be controlled is displayed in Figure 10.

The attendance of both vehicles in marginal points of their movements is checked by sensors indicating these locations on both sides of their runways - in case of AGV 2 at the conveyors (S2b, S2c) as well as at the transship center (S2a) and in case of AGV 1 at the transship center (S1b) as well as at the robot (S1a).

The place $p_{1}$ represents AGV2 being right, while $p_{4}$ represents AGV 2 being left. The place $p_{5}$ represents AGV1 being left, while $p_{7}$ indicates AGV 1 being right. 
The place $p_{6}$ denotes activities of the robot gripper. Unfortunately, there are unmeasurable/unobservable places $\left\{p_{2}, p_{3}, p_{8}, p_{9}\right\}$ in the PN model. They represent states of the unmeasurable/unobservable movements of AGVs on their runways. The place $p_{9}$ ensures repeating the working cycle.

The transition $t_{1}$ represents a start of the whole process. The sensors S2b, S2c correspond, respectively, to $t_{2}, t_{3}$. Sensors S2ab, S2ac correspond, respectively, to transitions $t_{4}, t_{5}$ depending on the fact whether AGV 2 arrives from S2b or from $\mathrm{S} 2 \mathrm{c}$. The transition $t_{6}$ presents the sensor S1a, $t_{7}$ denotes the sensor Spl of placing parts on the pressure plate, $t_{8}$ denotes the sensor S1b. Finally, $t_{9}$ is getting to be practically uncontrollable because of unobservable places $p_{8}, p_{9}$ - it has only deterministic information that $\mathrm{AGV} 2$ is left while information from $p_{8}, p_{9}$ being important for its firing is nondeterministic.

Moreover, while AGV 1 has an ambiguity consisting in $p_{1}$ (to fire either $t_{2}$ before $t_{3}$ or contrariwise) in AGV 2 no such ambiguity occurs. These facts have to be taken into account too.

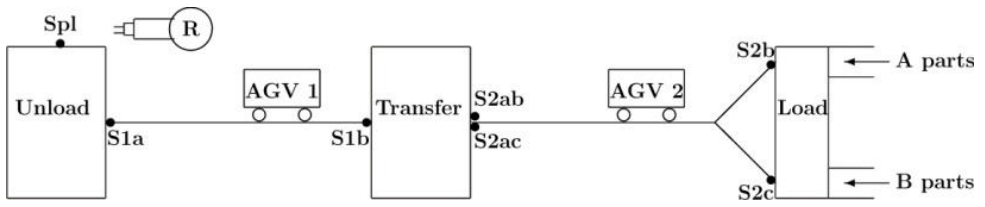

Figure 9

The rough scheme of the real FMS

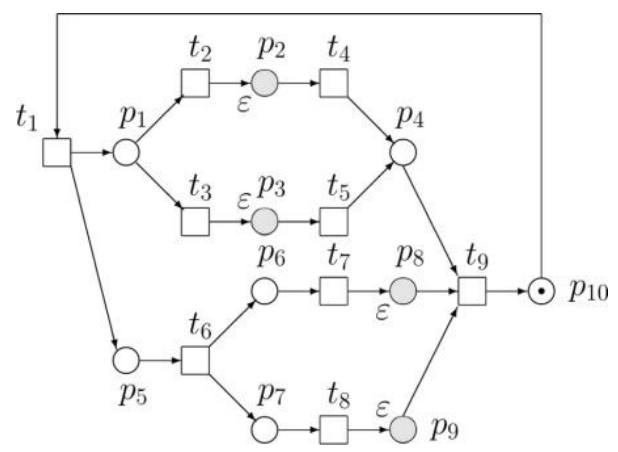

Figure 10

The PN model of the uncontrolled FMS

The RT of the PN model of such a system with controllable and observable transitions and measurable places is displayed in Figure 11.

Of course, building the IPN based model of DES itself is not sufficient. To ensure a desirable behavior of DES, it is necessary to synthesize the control specification able to deal with the non-determinism without affecting the technological process. 
Consider that the system behavior under the control should be as direct as possible, it means without any unnecessary turns, but in keeping within the technological process, of course. In such a case, the proposed controller corresponding to demands is added as it is apparent in Figure 12. The system behavior corresponds to the RT displayed in Figure 13.

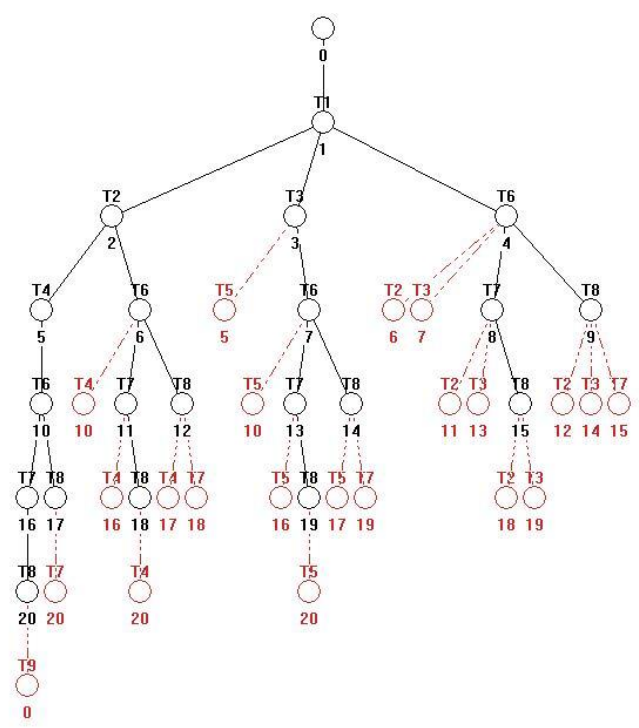

Figure 11

The RT corresponding to the PN model in Figure 10

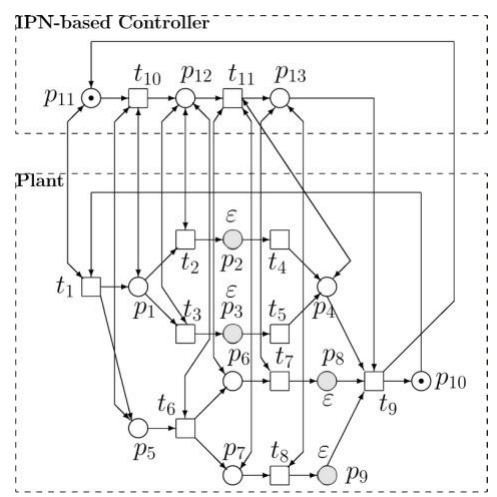

Figure 12

The IPN-based model of the controlled plant 


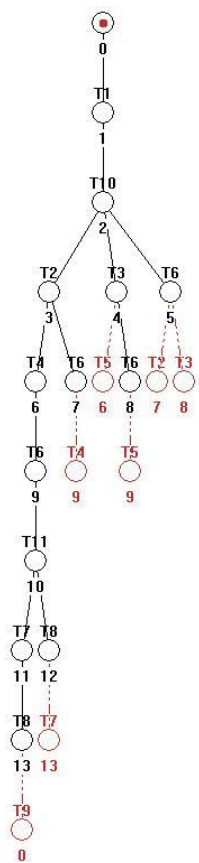

Figure 13

The RT corresponding to the IPN-based model of the controlled plant

\subsection{Example 2}

Let us introduce the example of DES where the situation is not so light. To illustrate problems, consider the real example of FMS consisting of two production lines L1, L2 producing mutually different parts $\mathrm{P} 1, \mathrm{P} 2$. The parts are assembled together into a final configuration $\mathrm{P} 1+\mathrm{P} 2$. The robot $\mathrm{R}$ machines parts in both lines. The line $\mathrm{L} 1$ is modeled by the subnet $\left\{p_{1}, p_{2}, p_{3} ; t_{1}, t_{2}, t_{3}\right\}$, while the line $\mathrm{L} 2$ by the subnet $\left\{p_{5}, p_{6}, p_{7} ; t_{4}, t_{5}, t_{6}\right\}$. The activities of the robot $\mathrm{R}$ are modeled by the subnet $\left\{p_{9}, p_{10}, t_{7}, t_{8}\right\}$, while those of the assembly process by the subnet $\left\{p_{4}, p_{8}, t_{9}\right\}$. Of course, the subnets are interconnected insomuch that it is impossible to strictly distinguish the confines among subnets. The PN model of the FMS is given in Figure 14. As we can see, firing $t_{8}$ is uncertain, because the transition $t_{8}$ is uncontrollable (it has the label $\varepsilon$ ). We do not know whether it, being enabled, will be fired in an actual state $k$ of the dynamics development or not. If not, $t_{8}$ as if did not exist $\left(u_{t_{8}}=0\right)$ and the PN structure has changed - the branch $p_{10} \stackrel{u_{t_{8}}}{\longrightarrow} p_{9}$ is as if dead, impassable, opaque. The RT of such PN structure is different from RT of the full structure together with $t_{8}$. In comparison with the RT of the full structure it is cropped - all branches labeled by $t_{8}$ are missing. Even, it contains a deadlock after the firing sequence $t_{7}, t_{4}, t_{5}, t_{6}$ starting from the initial state $\mathbf{x}_{0}$. Unfortunately, none of the trees can be introduced because of their size. 
Non-determinism consists in the displeasing fact that we do not know whether the enabled transition $t_{8}$ will be spontaneously fired - i.e. whether the process of the dynamics development will correctly continue, or not.

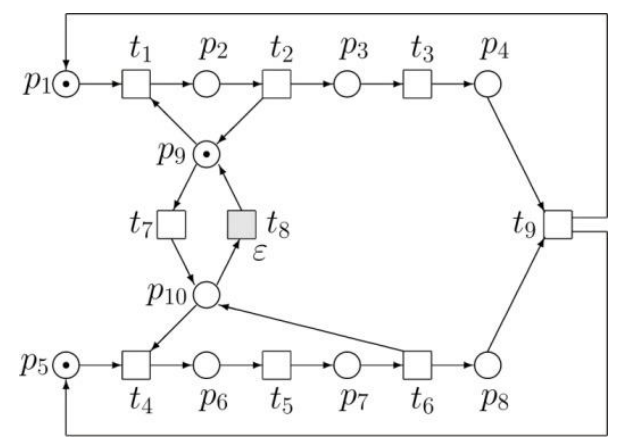

Figure 14

The P/T PN being the model of the FMS

\subsubsection{Using IPN-based Control}

Let us apply the IPN-based control of the plant given in Figure 14. Consider the same criterion for control as in the previous Example 1 (as direct as possible behavior of DES). Then, the PN model of the controlled plant is given in Figure 15. RT appurtenant to this model is displayed in Figure 17 up. The picture is rotated left (counter clockwise) in order to save space. However, such IPN-based controller needs not be alone. Other schemata can exist too. For example, a counter of production cycles can be added as it can be seen in Figure 16. It is represented by the place $p_{15}$. RT of such model is given in Figure 17 down. The picture is rotated left too because of saving space.

As we can see, the transition $t_{8}$ cannot be bypassed and it induces undesirable cycles in corresponding reachability trees $-\left\{\mathbf{x}_{4}, t_{8}, \mathbf{x}_{3}, t_{7}, \mathbf{x}_{4}\right\}$ in Figure 17 (up) and $\left\{\mathbf{x}_{15}, t_{8}, \mathbf{x}_{14}, t_{7}, \mathbf{x}_{15}\right\}$ in Figure 17 (down). It is necessary to say that there is no road to the satisfying solution in this case.

Now, let us investigate a situation when different transitions are uncontrollable or unobservable. Consider the structure of the IPN model given in Figure 18 with the uncontrollable transitions $t_{2}$ and $t_{6}$. In principle it is intrinsic e.g. in this case of DES when the upper branch $\left\{p_{1}, t_{1}, p_{2}, t_{2}, p_{3}, t_{3}, p_{4}\right\}$ with the fix resource $p_{9}$ (expressing the presence of a robot) represents a production line and the lower branch $\left\{p_{5}, t_{4}, p_{6}, t_{5}, p_{7}, t_{6}, p_{8}\right\}$ with the fix resource $p_{10}$ (expressing the presence of the same robot) represents another production line. Both lines are served by the same robot. While $p_{2}$ models an activity of a machine in the upper line, $\left\{p_{6}, t_{5}, p_{7}\right\}$ models an activity of another machine in the lower line. There the places $p_{13}, p_{14}$ are added to the IPN-based controller in order to define desired priorities of firing transitions - i.e. $t_{1}>t_{7}$ and $t_{8}>t_{9}$. 


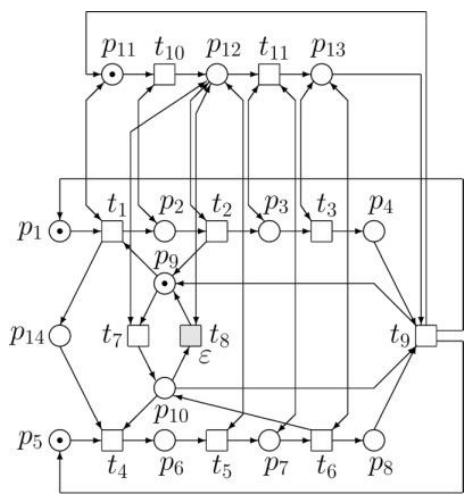

Figure 15

The scheme of the IPN-based control

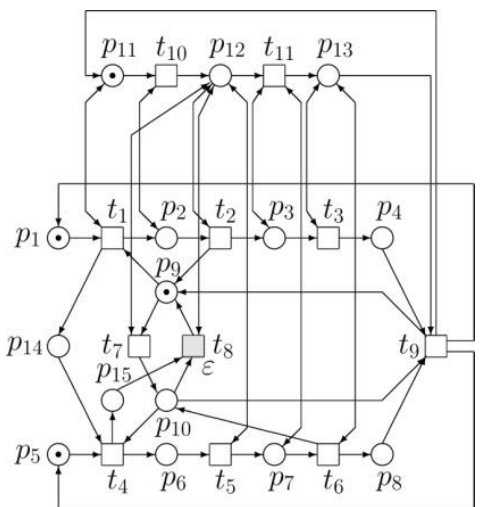

Figure 16

The scheme of the IPN-based control with the counter
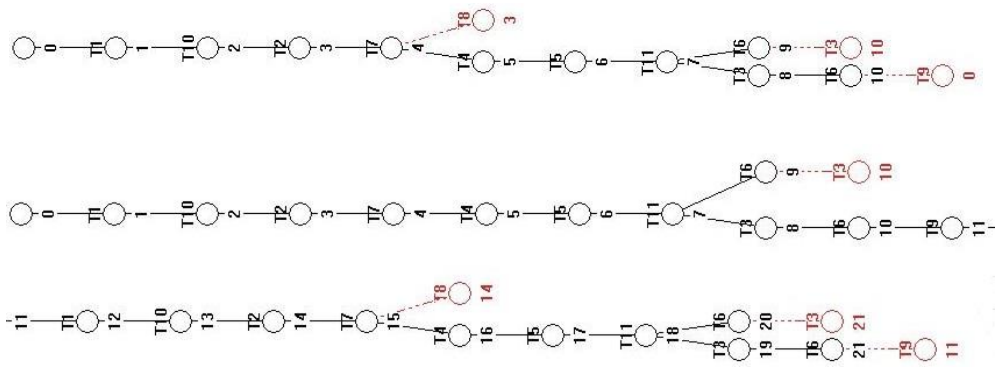

Figure 17

RT of the model in Figure 15 (up) and RT ( 2 parts) of the model in Figure 16 (down)

The global scheme of IPN-based control reflects a real situation, because finishing of the machining processes modeled by the PN subnets $\left\{t_{1}, p_{2}, t_{2}, p_{9}, t_{1}\right\}$ and $\left\{t_{4}\right.$, $\left.p_{6}, t_{5}, p_{7}, t_{6}, p_{10}, t_{4}\right\}$ is uncontrollable/unobservable because of the places $p_{2}$ and 
$\left\{p_{6}, p_{7}\right\}$ being practically unmeasurable/unobservable during machining. The RT of such controlled plant is given in Figure 19. As we can see, no cycles containing $t_{7}$ and $t_{8}$ exist there.

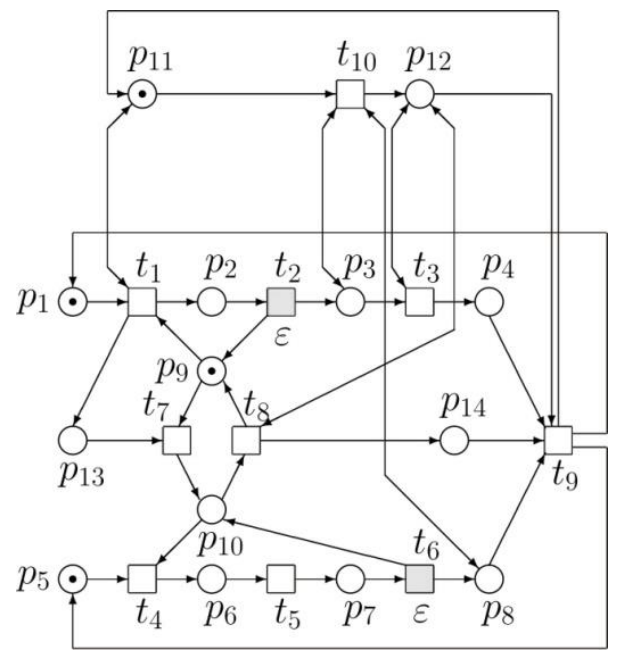

Figure 18

The IPN-based control of the system with uncontrollable transitions $t_{2}, t_{6}$

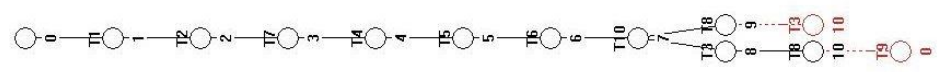

Figure 19

The RT of the system with uncontrollable transitions $t_{2}, t_{6}$

\subsection{The LbPN View on the Problem of Control}

Consider the LbPN model of a kind of DES, representing the cooperation of two jobs by means of resources $p_{5}, p_{6}$ needed for performing both jobs, given in Figure 20. Corresponding RT (provided that all transitions can be understood to be fired when they are enabled) is displayed in Figure 21. 


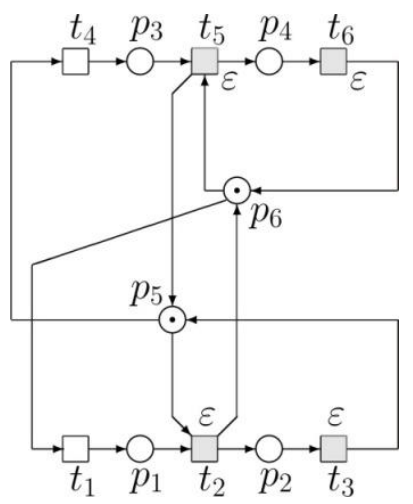

Figure 20

The LbPN model of DES

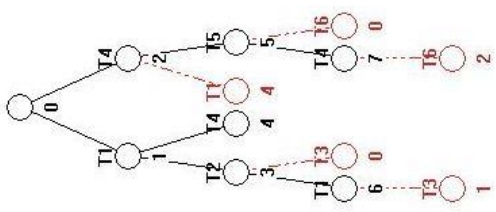

Figure 21

The corresponding RT

The resources must be available to satisfy the needs of both jobs. It means that the components $x_{p_{5}}, x_{p_{6}}$ of any state vector $\mathbf{x}_{k}, k=0,1, \ldots$, have to satisfy the following condition

$x_{p_{5}}+x_{p_{6}} \leq 2$

i.e. $\mathbf{L} \cdot \mathbf{x}_{k} \leq 2$, where $\mathbf{L}=\left(\begin{array}{llllll}0 & 0 & 0 & 0 & 1 & 1\end{array}\right)$ and $\mathbf{x}_{k}$ is an arbitrary reachable state. Then,

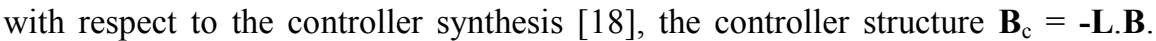
Hence, $\mathbf{B}_{\mathrm{c}}=\left(\begin{array}{llllll}-1 & 0 & 1 & -1 & 0 & 1\end{array}\right)$. Because $\mathbf{B}_{\mathrm{c}}=\mathbf{G}_{\mathrm{c}}{ }^{\mathrm{T}}-\mathbf{F}_{\mathrm{c}}$, where $\mathbf{G}_{\mathrm{c}}{ }^{\mathrm{T}}=\left(\begin{array}{llllll}0 & 0 & 1 & 0 & 0 & 1\end{array}\right)$ and

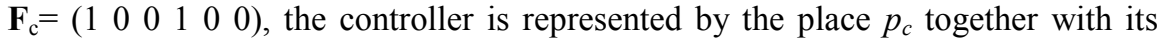
interconnections with the uncontrolled model - i.e. directed arcs from $p_{c}$ to transitions of the uncontrolled model by means of $\mathbf{F}_{\mathrm{c}}$, as well as from transitions of the uncontrolled model to $p_{c}$ by means of $\mathbf{G}_{\mathrm{c}}{ }^{\mathrm{T}}$. Consequently, the controlled LbPN model is given in Figure 22 and its RT is displayed in Figure 23. 


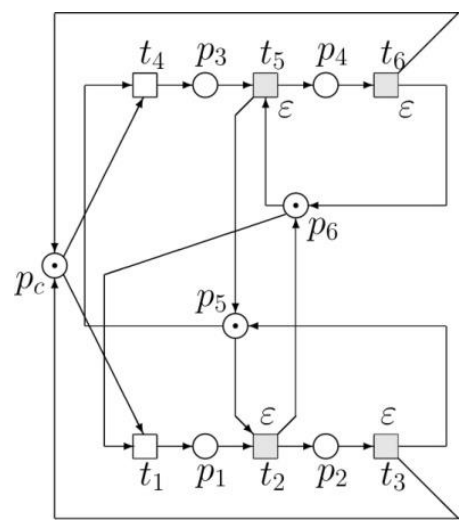

Figure 22

The LbPN model of DES

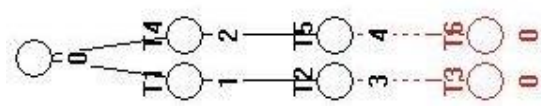

Figure 23

The RT of the LbPN model of DES

As we can see, the controller is able (i) to react on unobservable transitions $t_{3}, t_{6}$ when they are fired; (ii) to affect only the observable transitions $t_{1}, t_{4}$; (iii) to deal with the unobservable transitions $t_{2}, t_{5}$ when they are fired. Of course, the unobservable transitions cannot be either avoided or their influence eliminated. Moreover, both jobs are relatively autonomous - i.e. (i) $\mathbf{x}_{0} \stackrel{t_{1}}{\rightarrow} \mathbf{x}_{1} \stackrel{t_{2}(\varepsilon)}{\longrightarrow} \mathbf{x}_{3} \stackrel{t_{3}(\varepsilon)}{\longrightarrow} \mathbf{x}_{0}$ and (ii) $\mathbf{x}_{0} \stackrel{t_{4}}{\rightarrow} \mathbf{x}_{2} \stackrel{t_{5}(\varepsilon)}{\longrightarrow} \mathbf{x}_{4} \stackrel{t_{6}(\varepsilon)}{\longrightarrow} \mathbf{x}_{0}$.

\subsection{A Comment on Computational Complexity}

The computational complexity at using IPN and LbPN is nothing anomalous in comparison with P/T PN. Namely, the marking evolution of IPN and LbPN is the same like that of P/T PN and leads to computation of RT which may be a main source of problems concerning the computational complexity. Moreover, the structure of IPN and LbPN is prepared before the RT computation. The computational complexity of $\mathrm{P} / \mathrm{T} \mathrm{PN}$ is investigated in several papers concerning theoretical informatics - see e.g. [19]-[21]. For LbPN and IPN the total number of possible firing vectors is upper bounded by a polynomial function in $k$, i.e. $O\left(k^{b}\right)$, where $k$ is the length of the sequence of labels and $b$ is a parameter depending on the structure and the labeling function of the net. Namely $b=c(d-1)$, where $c$ is the number of nondeterministic labels and $d$ is the maximum number of transitions corresponding to a label in the net. 


\section{Conclusions}

This paper describes the possibility of how DES, with non-determinism, can be modeled and controlled by means of special kinds of PN, namely, IPN and LbPN. Such nets were defined and illustrative examples were introduced, in order to demonstrate their structure and dynamic behavior. The kernel of the paper is devoted, especially to the control synthesis of actual DES modeled, by means of these kinds of PN. It was shown, thru examples, that, in spite of non-determinism, real DES, in practice, can be controlled, albeit, with some limitations or restrictions.

\section{Acknowledgement}

This work was supported by the Slovak Grant Agency for Science VEGA under Grant No. 2/0029/17.

\section{References}

[1] C. A. Petri: Communication with Automata. Ph.D. Thesis. Technical University of Darmstadt, 1962, 128 pages (in German)

[2] J. L. Peterson: Petri Net Theory and the Modeling of Systems. Englewood Cliffs, NJ: Prentice-Hall, 1981

[3] T. Murata: Petri Nets: Properties, Analysis and Applications, Proceedings of the IEEE, Vol. 77, No. 4, 1989, pp. 541-580

[4] J. Desel, W. Reisig: Place/Transition Petri Nets. In: W. Reisig, G. Rozenberg (Eds.): Advances of Petri Nets, Lecture Notes in Computer Science, Vol. 1491, Springer, Heidelberg, 1998, pp. 122-173

[5] M. Silva: Half a century after Carl Adam Petri's Ph.D. thesis: A perspective on the field, Annual Reviews in Control, Vol. 37, 2013, pp. 191-219

[6] M. Silva: On the history of Discrete Event Systems, Annual Reviews in Control, Vol. 45, 2018, pp. 213-222

[7] A. Giua, M. Silva: Modeling, Analysis and Control of Discrete Event Systems: A Petri Net Perspective, In: $20^{\text {th }}$ IFAC World Congress, Toulouse, France, IFAC PapersOnLine 50-1, 2017, pp. 1772-1783

[8] Z. Ma, Y. Tong, Z. Li, A. Giua: Marking Estimation in Labelled Petri Nets by the Representative Marking Graph, In: $20^{\text {th }}$ IFAC World Congress, Toulouse, France, IFAC PapersOnLine 50-1, 2017, pp. 11175-11181

[9] M. P. Cabasino, C. N. Hadjicostis, C. Seatzu: Marking Observer in Labeled Petri Nets with Application to Supervisory Control, IEEE Transactions on Automatic Control, Vol. 62, No. 4, April 2017, pp. 1813-1824

[10] M. P. Cabasino, A. Giua, M. Pocci, C. Seatzu: Discrete Event Diagnosis Using Labeled Petri Nets. An Application to Manufacturing Systems, Control Engineering Practice, Vol. 19, 2011, pp. 989-1001 
[11] Z. Ma, Z. He, Z. Li, A. Giua: Design of Monitor-based Supervisors in Labelled Petri Nets, In: $14^{\text {th }}$ IFAC Workshop on Discrete Event Systems WODES, Sorrento, Italy, IFAC PapersOnLine 51-7, 2018, pp. 374-380

[12] M. P. Cabasino, C. N. Hadjicostis, C. Seatzu: State Feedback Control of Labeled Petri Nets with Uncertainty in the Initial Marking, In: $19^{\text {th }}$ International Conference on Emerging Technologies and Factory Automation ETFA, Barcelona, Spain, 2014, pp. 1-7

[13] A. Ramírez-Treviňo, I. Rivera-Rangel, E. López-Mellado: Observability of Discrete Event Systems Modeled by Interpreted Petri nets, IEEE Trans. on Robotics and Automation, Vol. 19, No. 4, 2003, pp. 557-565

[14] A. Giua: Analysis and Control of Petri Nets, In: DES School, Sorrento, Italy. Available in: http://schooldes2018.unisa.it/Giua_18wodes_school.pdf

[15] J. O. Moody, P. J. Antsaklis: Petri Net Supervisors for DES with Uncontrollable and Unobservable Transitions, IEEE Transactions on Automatic Control, Vol. 45, No. 3, March 2000, pp. 462-476

[16] Z. Achour, N. Rezg, X. Xie: Supervisory Controller of Petri Nets under Partial Observation, In: 7th International Workshop on Discrete Event Systems WODES, Reims, France, Sept. 22-24, 2004, IFAC Proceedings Vol. 37, No. 18, 2004, pp. 51-56

[17] A. Boussif, M. Ghazel, K. Klai: DPN-SOG: A Software Tool for Fault Diagnosis of Labeled Petri Nets Using the Semi-Symbolic Diagnoser, HAL Id: hal-01653191, Available on: https://hal.archives-ouvertes.fr/hal01653191, 2017, 14 pages

[18] F. Čapkovič: Timed and Hybrid Petri Nets at Solving Problems of Computational Intelligence. Computing and Informatics, Vol. 34, No. 4, 2015, pp. 746-778

[19] J. Esparza: Decidability and Complexity of Petri Net Problems - An Introduction. In: Reisig, W., Rozenberg, G. (Eds.): Lectures on Petri Nets I: Basic Models, Advances in Petri Nets, Springer, 1998, pp. 374-428

[20] H.-C. Yen: Introduction to Petri Net Theory. In: Recent Advances in Formal Languages and Applications. Book series Studies in Computational Intelligence (SCI), Vol. 25, Springer, 2006, pp. 343-373

[21] L. Li, C. Hadjicostis: Minimum Initial Marking Estimation in Labeled Petri Nets. In: Proceedings of the American Control Conference - ACC'2009, St. Louis, USA, paper FrB12.2, 2009, pp. 5000-5005 